\title{
Development of ANN Model for the Prediction of VIV Fatigue Damage of Top-tensioned Riser
}

\author{
Eileen Wee Chin Wong ${ }^{1}$, Han Suk Choi ${ }^{2}$, Do Kyun Kim ${ }^{2,3}$, and Fakhruldin Mohd Hashim ${ }^{1}$ \\ ${ }^{1}$ Department of Mechanical Engineering, Universiti Teknologi PETRONAS, Seri Iskandar, Malaysia \\ ${ }^{2}$ Graduate Institute of Ferrous Technology, POSTECH, Pohang, Republic of Korea \\ ${ }^{3}$ Department of Civil and Environmental Engineering, Universiti Teknologi PETRONAS, Seri \\ Iskandar, Malaysia
}

\begin{abstract}
Marine riser experiences vortex-induced vibration (VIV) caused by current, leading to fatigue damage if VIV is not considered in design of riser. Estimation of VIV fatigue damage is essential in designing feasible and operable riser. A simplified approach for predicting fatigue damage is required to reduce the computation time to analyze the fatigue damage. This study aims to explore the applicability of artificial neural network (ANN) approach in developing top-tensioned riser fatigue damage prediction model. A total of 2100 riser model is generated with different combination of four main input parameters: riser outer diameter, wall thickness, top tension and uniform current velocity. The modal analysis is performed using OrcaFlex and VIV fatigue damage of the riser is computed using SHEAR7. The four input parameters and corresponding fatigue damage results make up the database for training a 2-layer neural network. Weight and bias values acquired from the training of ANN are used to develop the VIV fatigue damage prediction model of the riser. The results show ANN approach is suitable for prediction of the riser fatigue damage due to VIV. The proposed approach requires further refinements and extension to more input features to improve the accuracy and usefulness of the developed prediction model.
\end{abstract}

\section{Introduction}

Marine risers play integral part in offshore exploration and production. They serve several purposes, with some function as drilling risers to guide drilling and transport drilling fluid for well control, and some operate as production risers to transport hydrocarbon from seabed to platform. Fatigue design of riser is important to ensure safety of the riser. Vortexinduced vibration is one of the vital factors causing fatigue damage of the riser. When fluid flows over the circular cylinder, vortex shedding occurs which causes pressure differential around the cylinder and induces forces on the cylinder, causing it to vibrate. When the cylinder vibrates at or near to the frequency of vortex shedding, lock-in phenomenon happens, which excites very high response amplitude of vibration of the riser and causes the fatigue failure of the riser structure.

Current is the main factor contributing to VIV fatigue damage of the riser. The higher the current speed, higher mode of vibration is excited, leading to greater amplitude of 
vibration and hence greater fatigue damage [1]. Gao et al. [2] adopted a simplified vibration model with the characteristics of SCR and found that maximum fatigue damage increases as current velocity increases. In the following year, Gao et al. [3] discovered that the number of modes, which contribute to vibration of the riser, increases when current velocity increases. As more oil and gas field developments have been shifted to deepwater regions progressively, VIV has become a subject undergoing intense studies over the past few decades. A comprehensive review of the fundamental discoveries with respect to VIV is well beyond the scope of this paper and can be found in Williamson and Govardhan [4].

VIV analysis is important part in design procedure of marine riser [5]. Four methods are generally used to assess the riser response and fatigue damage associated with VIV, as highlighted in DNV-OS-F201 [6]. During the preliminary study of VIV design of riser, DNV [6] recommended usage of simplified approach with the assumption of undisturbed current velocities being exerted to the riser model. Vibration amplitude and the corresponding stress range of dominant mode are computed for the given current velocity. Fatigue damage is calculated using appropriate S-N curve correspond to the type of material of the riser pipe $[6,7]$. In the case of significant VIV fatigue damage being diagnosed, more detailed analysis should be performed using laboratory testing, semi empirical multi-modal response analysis or computational fluid dynamic approach.

Experimental or small-scale modal test is the most direct method to understand VIV behavior of the riser. Many VIV experiment were carried out in the past. Some of the meaningful experiments are Norwegian Deepwater Program [8] and Deepstar JIP [9]. These experiments produce database that are used to develop and validate analytical tools and software. Several computer models are developed to predict the VIV behavior of riser in the flow region where modal testing cannot be performed. Many state-of-practice software were developed using the empirical or semi-empirical data obtained from modal test, such as SHEAR7 [10], VIVANA [11] and VIVA [12]. These softwares perform multimodal response analysis based on empirical hydrodynamic coefficient obtained from measurement data of rigid cylinders experiencing VIV and compute VIV response using frequency domain approach. A number of VIV studies have been conducted by frequency domain based softwares [13-15].

Computational fluid dynamic (CFD) approach is also another popular option to study the VIV behavior of riser. CFD applies the solution of the Navier-Stokes equation. Strip theory is commonly used where fluid flow solutions represented in term of strips are computed on series of two-dimensional (2D) planes distributed over the riser length and combined with riser structural model [16]. However, it is unable to reproduce the actual three-dimensional (3D) flow and certain geometries [17]. Huang et al. [18] proved the feasibility of using the CFD time domain simulation approach and Chimera technique in assessing deepwater riser VIV. Huang also conducted 3D CFD simulation using FiniteAnalytic Navier-Stokes (FANS) code based on the local-analytic-based discretization procedure.

Despites of the considerable advances made in estimating VIV behavior of the marine risers, most of these methods have complicated and tedious calculation. Furthermore, they are computationally expensive. Hence, researchers have been on the quest to search for method to reduce the computational cost. One of the methods is combining finite element method and artificial neural network (ANN) [15]. By using the result of response analysis of a structure in FEM, an ANN is trained to predict the response for future load. Guarize et al. [20] have presented a highly accurate ANN to predict top tension dynamic response of mooring line. Christiansen et al. [21] applied similar approach to assess full fatigue life of mooring line. Both studies show drastic reduction of the simulation time using ANN. Chaves et al. [22] have adopted the same approach for calculating fatigue of flexible pipes. They proved that hybrid ANN-FEA approach decreases the computation time of 
conventional full FEA method by factor of 22. Quéau et al. [23] demonstrated the use of ANN to develop framework to approximate maximum dynamic stress range in steel catenary riser.

The past studies of application of ANN for different structures have shown great accuracy in prediction and reduction in time. Therefore, the aim of this paper is to explore the application of ANN to predict the fatigue damage of a top-tensioned riser due to VIV. An ANN is trained using four selected input features and fatigue damage result obtained from fatigue analysis using OrcaFlex and SHEAR7. The ANN recognizes the relationship and generates a mathematical model to relate the input parameters to the VIV fatigue damage of the riser. The mathematical model provides simplified approach to predict fatigue damage of riser.

\section{Top-tensioned Riser Model}

This study is limited to 2D top-tensioned riser fatigue damage subjected to uniform current. Variation in wave and wind motion, flow rate of the contents, coating and material properties are not taken into consideration in this study but will be explored in future study. A linear soil model is used. With these assumption, four variables are identified as pipe outer diameter, wall thickness, applied top tension, and uniform current speed. To explore the applicability of ANN in predicting fatigue damage rate of riser, a set of training data with both input variables and resulting fatigue damage is required to train ANN. Fig. 1 shows the flowchart of the methodology to develop the simplified approach to predict fatigue damage of riser.

A simple top-tensioned riser is modelled in OrcaFlex based on the riser data by Barltrop [24], in accordance to design codes ISO 13624-2 [25]. OrcaFlex is a finite element program specific for use in marine dynamics. Analysis is performed for bare riser with properties listed in Table 1 as the base model. In the model, as portrayed in Fig. 2, the coordinate system is defined as such: $\mathrm{x}$-axis is horizontal axis along the riser plane parallel to the the seabed; y-axis is perpendicular to the riser plane; z-axis is the vertical axis along the riser plane representing the water depth. The water depth is $500 \mathrm{~m}$. Only the riser is modelled because riser is the focus of study in the VIV fatigue analysis. The vessel motion is assumed to be fixed, hence no translational motion in $\mathrm{x}$ and $\mathrm{y}$ direction at the top end of the riser. The top end of the riser is only allowed to move axially with constant top tension applied on it. The riser is anchored to the seabed at the bottom end. The fluid flow inside the riser is assumed to be uniform, which means no slugging occurrence to be observed. The current is assumed to be unidirectional throughout the water depth.

Table 1. Bare riser model properties.

\begin{tabular}{|l|c|}
\hline Riser length & $530 \mathrm{~m}$ \\
\hline Riser material & Steel \\
\hline Material density & 7.85 tonne $/ \mathrm{m} 3$ \\
\hline Internal fluid density & 0.9 tonne $/ \mathrm{m} 3$ \\
\hline Internal fluid pressure & $6.895 \mathrm{MPa}$ \\
\hline
\end{tabular}




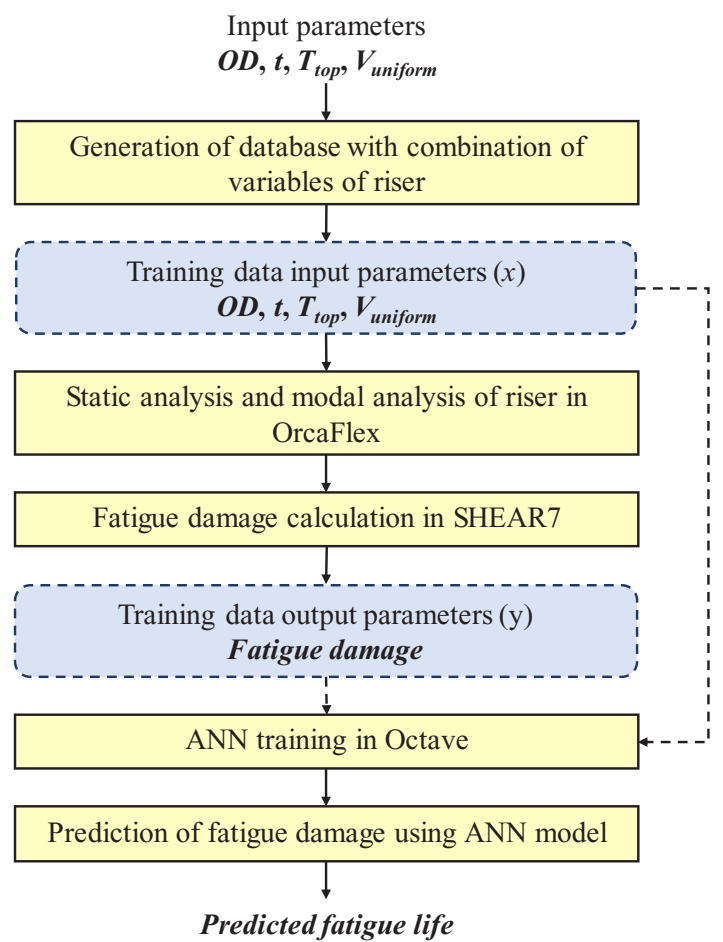

Fig. 1. Flowchart of methodology to obtain fatigue damage prediction model.

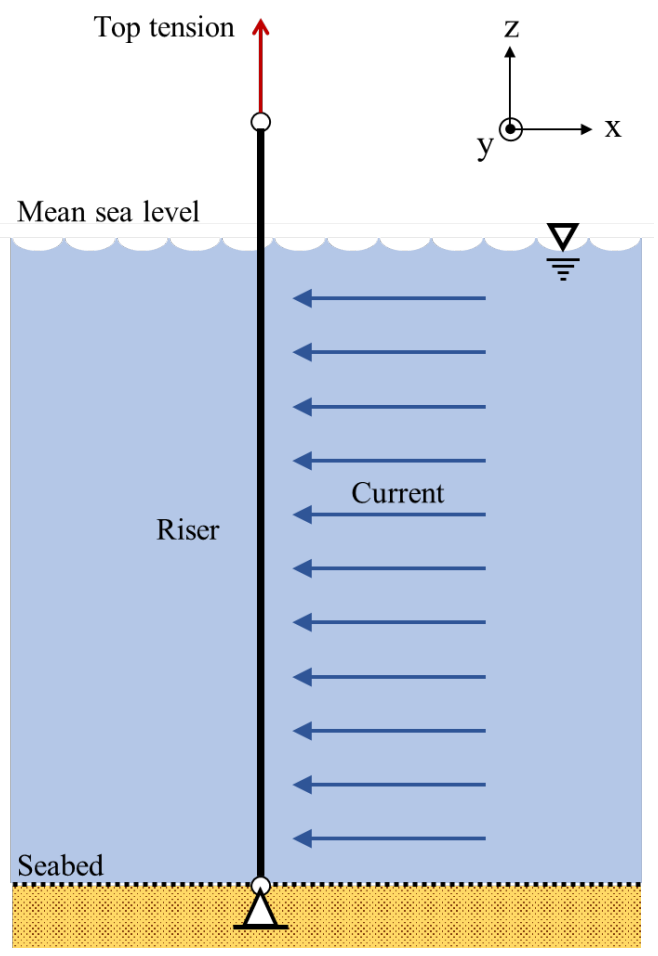

Fig. 2. Top-tensioned riser model. 


\section{Generation of Riser Input Variable Database}

The minimum and maximum values for outer diameter, wall thickness, top tension and uniform current velocity are determined and form the boundary for the design space of toptensioned riser. The extreme values of the input parameters are presented in Table 2. The values of each parameters are varied and combined to form cases of riser model with different input values. Cases that produce unstable equilibrium or cases with negative tension at the bottom end of the riser are eliminated since these are unrealistic cases. Hence, a total of 2100 cases are generated from different combination of the four input parameters. Static analysis and modal analysis of the riser are performed in OrcaFlex to determine the static equilibrium and natural frequency of the riser. The natural frequency of the riser is used as input in SHEAR7. SHEAR7 is used to perform frequency domain calculation of the fatigue damage of the riser based on mode superposition. The maximum fatigue damage of riser in each case are recorded in the database as the output y data to be used as training set together with four input parameters, $\mathrm{x}$, for the ANN.

Table 2. Maximum and minimum value of input parameters.

\begin{tabular}{|l|c|c|}
\hline Input parameter & Minimum value & Maximum value \\
\hline Outer diameter, OD $(\mathrm{m})$ & 0.32385 & 0.762 \\
\hline Wall thickness, $\mathrm{t}(\mathrm{mm})$ & 7.14 & 19.05 \\
\hline Top tension, Ttop $(\mathrm{kN})$ & 1000 & 1800 \\
\hline $\begin{array}{l}\text { Uniform current valocity, } \\
\text { Vuniform }(\mathrm{m} / \mathrm{s})\end{array}$ & 0.2 & 1.0 \\
\hline
\end{tabular}

\section{Artificial Neural Network}

Artificial neural network (ANN) is a type of machine learning model that recognizes complex relationships between groups of inputs and corresponding output. It is an algorithm created to try to mimic brain in recognizing patterns. It becomes state-of-art technique for many applications, such as pattern recognition, data classification, forecasting, optimization etc. ANN can approximate closely any nonlinear mathematical function, with certain error depending on the ANN architectures and its hyperparameter.

\subsection{ANN Architecture}

A typical two-layer neural network, also known as shallow neural network, is shown in Fig. 3. It consists of three layers. The first layer is input features and usually not counted as a layer. The second layer is known as hidden layer, which contributes non-linearity in the ANN model. The last layer is output layer that generates result. These three layers are interconnected by mathematical elements known as artificial neurons. Each neuron carries a weight which controls the function mapping from layer to layer. Every node of a layer are connected to every one of the nodes in adjacent layer. The neurons are fed with inputs, compute weighted summation and implement activation function and activate the nodes in subsequent layer. 


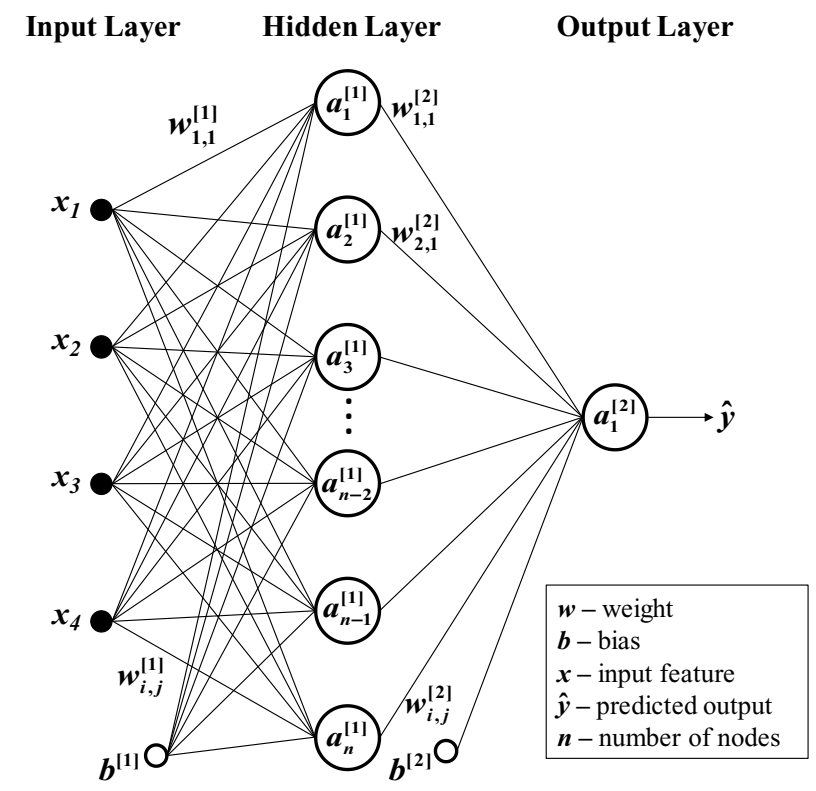

Fig. 3. Architecture of 2-layered neural network.

Multiple layer neural network, which is known as deep neural network, can also be used to solve more complicated problems. Deep neural network consists of multiple hidden layers to perform more complicated mapping and learning. Both number of hidden layers and number of nodes in each layer are hyperparameters that need to be determined to capture the relationships accurately without overfitting. Too many nodes in hidden layer may leads to overfitting causing the network fail to generalize to new test examples. Besides that, it will increase the computational time to train ANN. Generally, one hidden layer is sufficient for most application.

\subsection{Structure of ANN}

In this study, 2-layer neural network shown in Fig. 3 is used since the use of single hidden layer is sufficient for most application and recommended for pilot study. Four input features are identified as pipe outer diameter, wall thickness, applied top tension and uniform current speed. A total of 100 hidden units are used in the hidden layer and a single output unit for computing the fatigue damage of riser is used.

Each connection in ANN has a weight, $w_{i, j}$. In the first hidden layer, the neuron at position $j$ receives value of $x_{i}$ from input feature $i$ weighted by $w_{i, j}$ and calculates the weighted sum of all the input features from input layer. The resulting sum is added with a bias unit $b$. The summation is expressed in (1) below.

$$
z_{j}^{[l]}=b^{[l]}+\sum_{i=1}^{n} w_{i, j}^{[l]} x_{i}
$$

where $z_{j}$ is the resulting summation at node $j, n$ is the number of hidden units and $l$ is layer. For input layer, $l$ will be 0 and for subsequent hidden layers, $l$ will be $1,2,3 \ldots$ so on. 
Activation function is used to produce output. The output, $a_{j}$ is defined in (2). The output for layer $l$ will become input for adjacent layer $l+1$. The process of weighted summation and activation function implementation will be repeated till the output layer.

$$
a_{j}^{[l]}=\varphi\left(z_{j}^{[l]}\right)
$$

There are several types of activation function commonly used in ANN, which are linear function, sigmoid function, tanh function, rectified linear unit function etc. For this application, tanh function is chosen as activation function for hidden layer because it centers the data and works better than sigmoid function. Linear function is set for the output layer to provide a continuous output value. The tanh function is defined in (3) and linear function is given in (4).

$$
\begin{gathered}
\varphi(z)=\frac{e^{z}-e^{-z}}{e^{z}+e^{-z}} \\
\varphi(z)=z
\end{gathered}
$$

In general, the neuron in layer $l$ computes the weighted sum of input from previous layer $l-1$ and activation function is implemented to produce output. This output is sent as input to neuron in the next layer $l+1$. This builds up the complete ANN.

\subsection{ANN Training}

Training of ANN is required to obtain optimal weight matrix $\mathrm{W}$ for accurate prediction of output. Optimization of the weight value can be performed by minimizing the cost function of the entire training data. The cost function, $\mathrm{J}$ used in this neural network is mean squared error function. It is defined as below

$$
J(w, b)=\frac{1}{2 m}\left[\sum_{i=1}^{m}\left(\hat{y}_{i}-y_{i}\right)^{2}+\lambda \sum_{j=1}^{n} w_{j}^{2}\right]
$$

where $\hat{y}$ is the predicted output, $\mathrm{m}$ is the number of training data, $\lambda$ is the regularization parameter and $i$ is training set number. L2 regularization is used in the training algorithm to reduce model overfitting. The regularization parameter reduces values of weight and makes the model less prone to overfitting.

Since ANN is a non-linear method, it does not have direct approach to compute the parameters with minimum cost function. To minimize the cost function, optimization algorithm is required. In this study, gradient descent algorithm is applied. Gradient descent is an iterative optimization algorithm that take steps repeatedly in the direction of gradient at current point. In each iteration, derivative of the cost function with respect to weight is computed. Weight value is updated in direction of downward gradient. The process is iterated until the cost function reaches the lowest point.

Before the gradient descent is employed, the training data input values need to be normalized to speed up the learning algorithm. The values of weight are randomly initialized using Xavier initialization [26]. The initial weight values are random value multiplied by scale defined below.

$$
\varepsilon_{\text {init }}=\sqrt{\frac{1}{\text { dimension of previous layer }}}
$$

The gradient descent algorithm consists of three main steps. First step of the algorithm is forward propagation. After weight initialization, in each iteration, the neurons in each 
layer compute the activation function of the weighted sum of the all inputs with the initialized weight values. As mentioned in the previous section, tanh function is used as activation function in hidden layer and linear function is used to obtain the output value at the output layer. At the output node, the cost function of all the training data is computed using (5).

In the second step, backpropagation is performed. The difference between the predicted output and actual y data is compared and error is calculated as $d z$. The $d z$ value is then used to compute derivative of cost function with respect to weight and bias, which are $d w$ and $d b$ respectively. The $d z, d w$ and $d b$ for each layer are calculated. In the last step, weight and bias values are updated simultaneously using the calculated derivatives. Equation (7) shows the calculation of new weight and bias at each iteration,

$$
\begin{aligned}
& w_{j}^{[l]}=w_{j}^{[l]}-\alpha \frac{d J(w, b)}{d w_{j}^{[l]}} \\
& b^{[l]}=b^{[l]}-\alpha \frac{d J(w, b)}{d b^{[l]}}
\end{aligned}
$$

where $\alpha$ is learning rate. The learning rate controls the size of the step taken in the direction of descent. If the learning rate is too small, the learning algorithm takes long time to compute. If high learning rate is used, the optimization of cost function is less likely to converge. Hence, it is recommended to use adaptive learning rate.

Fig. 4 portrays the flowchart of complete iterative process of training neural network using gradient descent algorithm in Octave. The calculation is done in vectorized approach. The capitalized parameters such as $X, Y, W, A$ and $Z$ represent matrix form of parameters $x$, $y, w, a$ and $z$. Vectorized algorithm increases the speed of training the neural network. The acquired weight and bias values at the end of training are used to build fatigue damage prediction model.

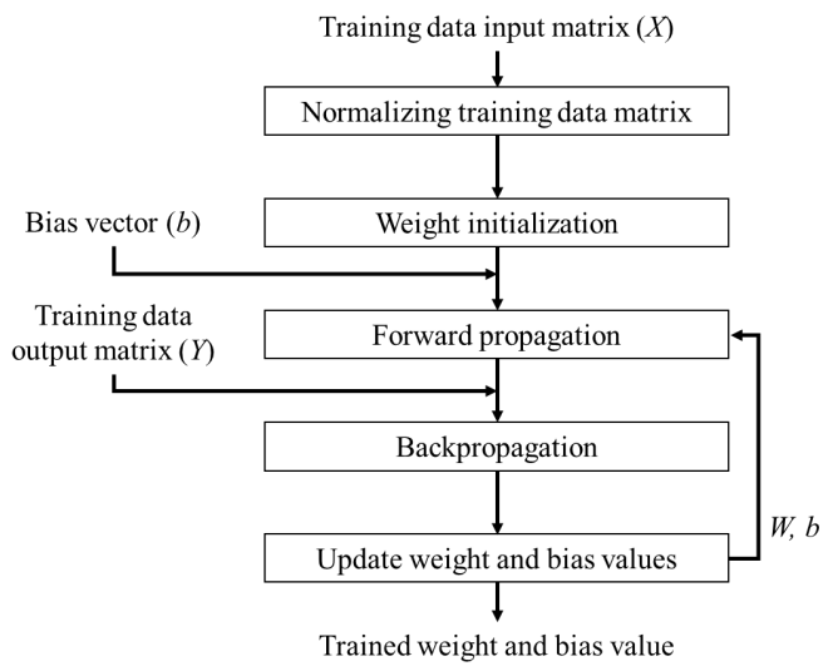

Fig. 4. Flowchart of training ANN using Octave.

\section{Result and Discussion}

To define and validate ANN model, the database is divided into three sets: training set, cross-validation $(\mathrm{CV})$ set and test set. The training set is used to train the ANN and 
determine the weight and bias parameters to build fatigue damage prediction model of riser. The CV set is used to tune and pick ANN model with appropriate hyperparameter, such as number of hidden unit, that can produce the lowest mean-squared error. Test set is used to evaluate the performance of the ANN model. From the ANN model tuning with CV set, ANN model with 100 hidden units produces the lowest mean squared error. Hence, ANN with 100 hidden units model is selected. Fig. 5 and 6 illustrate the fatigue damage rate predicted by presented ANN model compared to fatigue damage rate computed from SHEAR7.
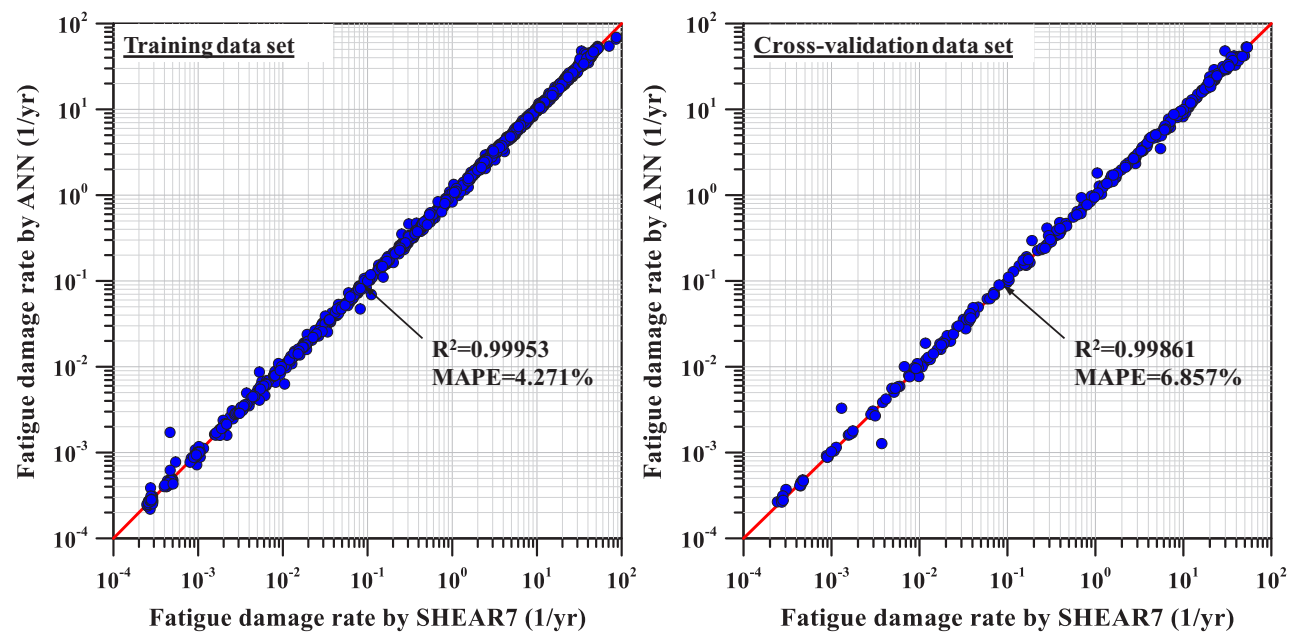

Fig. 5. Comparison of fatigue damage by ANN and SHEAR7 for training and validation data.

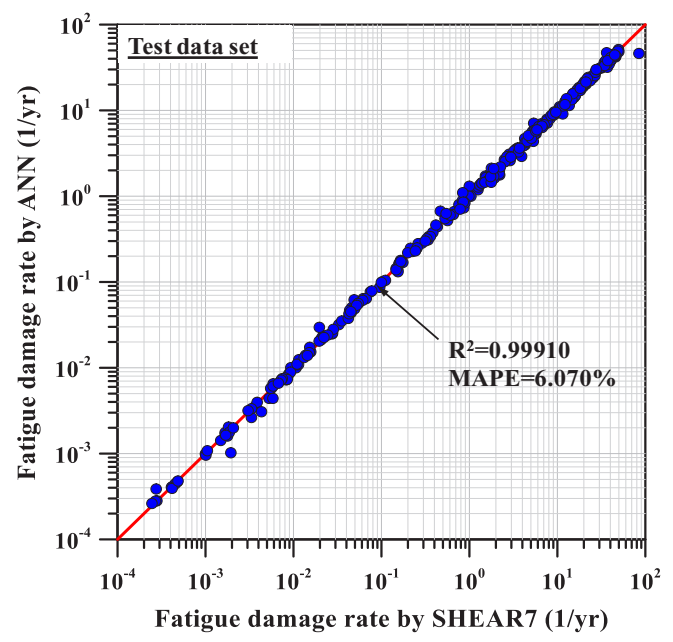

Fig. 6. Comparison of fatigue damage by ANN and SHEAR7 for test data.

Coefficient of determination $\left(\mathrm{R}^{2}\right)$ and mean absolute percentage error (MAPE) are used to evaluate the performance of the presented ANN model. $\mathrm{R}^{2}$ is statistical measure of the ability of the presented model predicts the outcome. The value of $R^{2}$ ranges from 0 to 1 . The closer the $\mathrm{R}^{2}$ value to 1 , the better the model fits with the data. MAPE measures the error in term of percentage. Table 3 shows the prediction performance of the presented 
ANN model for all the three datasets. The $\mathrm{R}^{2}$ value of test set is close to 1 and the MAPE of the test set is considerably small. These shows that ANN is capable to learn from the data and predict well the VIV fatigue damage. Therefore, the proposed ANN model is suitable to be used to build VIV fatigue damage prediction model of riser.

Table 3. Prediction performance of ANN

\begin{tabular}{|l|c|c|c|}
\cline { 2 - 4 } \multicolumn{1}{c|}{} & Training set & CV set & Test set \\
\hline $\mathrm{R}^{2}$ & 0.99953 & 0.99861 & 0.99910 \\
\hline MAPE & $4.271 \%$ & $6.857 \%$ & $6.070 \%$ \\
\hline
\end{tabular}

\section{Conclusion}

A numerical study to explore the applicability of artificial neural network for estimating VIV fatigue damage of top-tensioned riser was conducted in this paper. OrcaFlex and SHEAR7 were used to build the riser model and compute the fatigue damage of riser. The results generate database of 2100 cases with four input variables (outer diameter, wall thickness, top tension, uniform current velocity) and corresponding fatigue damage rate to be used to train the ANN. The obtained weight and bias from training of ANN model were used to build fatigue damage prediction model. From validation result using test set, the error produced by the presented ANN model is $6.07 \%$, which is considerably low. Therefore, the accuracy of ANN model is acceptable. Overall, ANN approach is well-suited for predicting fatigue damage of top-tensioned riser.

The variation in other riser parameter and current will be explored and included in the ANN model to improve the usefulness of the prediction model. More advanced algorithm will be investigated to improve the speed and accuracy of training the neural network. Different neural network architecture will be examined to search for prediction model with the best performance.

\section{Acknowledgements}

This study was undertaken at Ocean and Ship Technology (OST) research group at Universiti Teknologi PETRONAS. This research was supported by the Technology Innovation Program (Grant No.: 10053121 and 10051279) funded by the Ministry of Trade, Industry \& Energy (MI, Korea) and YUTP Grant (0153AA-E60, Malaysia). The authors would also like to thank for the great support of POSTECH, POSCO, and Daewoo E \& C, Republic of Korea.

\section{References}

1. D.K. Kim, E.W.C. Wong, E.B. Lee, S.Y. Yu, and Y.T. Kim, "A method for the empirical formulation of current profile," Ships and Offshore Structures, In-press (https://doi.org/10.1080/17445302.2018.1488340).

2. Y. Gao, Z. Zong, L. Zhou, and J. Cao, "Analysis of vortex induced vibration fatigue damage of steel catenary riser," Chinese J. of Ship Research, 5, no.5, pp. 54-63, 2010.

3. Y. Gao, Z. Zong, and L. Sun, "Numerical prediction of fatigue damage in steel catenary riser due to vortex-induced vibration," J. Hydrodyn., vol. 23, no. 2, pp. 154-163, Apr. 2011. 
4. R.H.J. Willden and J.M.R. Graham, "CFD simulations of the vortex-induced vibrations of model riser pipes," in Proc. 24th Int. Conf. OMAE, Halkidiki, Greece, Jun. 2005.

5. DNV, "Riser Fatigue," Det Norske Veritas, Recommended Practice F204, 2010.

6. DNV, "Dynamic Risers," Det Norske Veritas, Offshore Standard F201, 2010.

7. R.D. Blevins, Flow-induced vibrations, Van Nostrand Reinhold Company, 2001.

8. A.D. Trim, H. Braaten, H. Lie, and M.A. Tognarelli, "Experimental investigation of vortexinduced vibration of long marine risers," J. Fluids Struct., vol. 21, pp. 335-361, 2005.

9. V. Jhingran, V. Jaiswal, and J.K. Vandiver, "Spatial Variation of Drag on Long Cylinders in Sheared Flow," in Proc. 27th Int. Conf. OMAE, Estoril, Portugal, Jun. 2008.

10. J.K. Vandiver, "User guide for SHEAR7," Massachusetts Institute of Technology, 1999.

11. C.M. Larsen, K. Vikestad, R. Yttervik, E. Passano, and G.S. Baarholm, "Theory Manual," MRINTEK 51310.01, 2000.

12. M.S. Triantafyllou, "VIVA extended user's manual," Massachusetts Institute of Technology, 2003.

13. K.S. Park, Y.T. Kim, D.K. Kim, S.Y. Yu, and H.S. Choi, "A new method for strake configuration design of steel catenary risers," Ships and Offshore Structures, Vol. 11, No. 4, pp. 385-404, 2016.

14. D.K. Kim, A. Incecik, H.S. Choi, E.W.C. Wong, S.Y. Yu, and K.S. Park, "A simplified method to predict fatigue damage of offshore riser subjected to vortex-induced vibration by adopting current index concept," Ocean Engineering, Vol. 157, pp. 401-411, 2018.

15. Y.T. Kim, D.K. Kim, H.S. Choi, S.Y. Yu, and K.S. Park, "Fatigue performance of deepwater steel catenary riser considering nonlinear soil effect," Structural Engineering and Mechanics, Vol. 61, No. 6, pp. 737-746, 2017.

16. K.W. Schulz and T.S. Meling, "VIV analysis of a riser subjected to step and multi-directional currents," in Proc. 24th Int. Conf. OMAE, Halkidiki, Greece, Jun. 2005.

17. S. Holmes, Y. Constantinides and O.W. Oakley, Jr., "Simulation of riser VIV using fully three dimensional CFD simulations," in Proc. 25th Int. Conf. OMAE, Hamburg, Germany, Jun. 2006.

18. Z.M. Huang, "CFD simulation of riser VIV," Ph.D. dissertation, Dept. Ocean Eng., Texas A\&M Univ., 2011.

19. H. Adeli, "Neural networks in civil engineering: 1989-2000," Comput-Aided Civ. Inf., vol. 16, no. 2 , pp. 126-142, Mar. 2001.

20. R. Guarize, N.A.F. Matos, L.V.S. Sagrilo, and E.C.P. Lima, "Neural networks in the dynamic response analysis of slender marine structures," Appl. Ocean Res., vol. 29, no. 4, pp. 191-198, Nov. 2007.

21. N.H. Christiansen, P.E.T. Voie, J. Høgsberg, and N. Sødahl, "Efficient mooring line fatigue analysis using a hybrid method time domain simulation scheme," in Proc. 32nd Int. Conf. OMAE, Nantes, France, Jun. 2013.

22. V. Chaves, V.R.M. Silve, L.V.S. Sagrilo, and M.A. Vignoles, "Artificial neural networks applied to flexible pipes fatigue calculations," in Proc. 34th Int. Conf. OMAE, Newfoundland, Canada, May-Jun. 2015.

23. L.M. Quéau, M. Kimiaei, and M.F. Randolph, "Approximation of the maximum dynamic stress range in steel catenary risers using artificial neural networks," Eng. Struct., vol. 92, pp. 172-185, 2015.

24. N.D.P. Barltrop, Floating Structures - A Guide for Design and Analysis, Volumes 2, 1998.

25. ISO, "Petroleum and natural gas industries-Drilling and production equipment -Part 2: Deepwater drilling riser methodologies, operations, and integrity technical report," International Organization for Standardization, 2009.

26. X. Glorot and Y. Bengio, "Understanding the difficulty of training deep feedforward neural networks", in Proc. 13th Int. Conf Aistats, 2010. 